\title{
Une forme sévère de lissencéphalie est liée à des délétions du chromosome 17
}

Les lissencéphalies (ou agyries) sont des affections neurologiques de haute gravité, provenant d'une insuffisance de la migration neuronale chez le fotus entre 3 et 5 mois, et aboutissant à l'absence de circonvolutions cérébrales. On en distingue différents types : dans le type I, on individualise le syndrome de Miller-Dieker $\left(\mathrm{n}^{\circ} 24720 \mathrm{du}\right.$ catalogue McKusick) [1], dont les traits caractéristiques incluent une dysmorphie faciale accusée, et une forme moins sévère avec dysmorphie légère ou absente. Dans le type II, à l'agyrie s'ajoutent des troubles cérébelleux et oculaires : c'est le syndrome de WalkerWarburg ou syndrome HARD (hydrocéphalie, agyrie, dysplasie rétinienne). Son hérédité est autosomique récessive ( $\left.\mathrm{n}^{\circ} 23667\right)$.

C'est dans le syndrome de MillerDieker que sont connues des anomalies chromosomiques, dont Dobyns et al. [2] viennent de donner une mise au point. Les auteurs ont réuni 25 familles dont certains membres remplissent les critères anatomocliniques du syndrome. Dans 22 d'entre elles, ils ont pu démontrer l'existence d'une délétion portant sur le bras court du chromosome 17 en 17p13.3. Dans 14 cas la délétion pouvait être reconnue cytologiquement alors que dans les 8 autres le caryotype paraissait normal et la délétion a été détectée grâce à l'emploi de sondes marquant le région. La taille des délétions ainsi mises en évidence était d'au moins $370 \mathrm{~kb}$. Il est possible que les trois malades qui n'en ont pas montré aient des délétions plus petites, mais une autre origine ne peut être exclue. Lorsqu'on examine l'origine des anomalies, on constate que les lésions apparues de novo sont de loin les plus fréquentes, 11 sur 14, alors que trois cas seulement sont familiaux, provenant de translocations équilibrées chez un parent ; ce sont ces formes qui avaient fait attribuer initialement à la maladie une transmission autosomique récessive.

Enfin, parmi les 11 sujets pour lesquels on a pu déterminer l'origine parentale du chromosome délété de novo, il s'agissait sept fois du chromosome 17 paternel et quatre fois du maternel. Il apparaît donc que le bras court du chromosome 17 porte une région critique pour la migration des cellules neuronales. La taille élevée des délétions indique qu'elles contiennent probablement plusieurs gènes, de manière comparable aux syndromes associés aux tumeurs de Wilms et au rétinoblastome sans pouvoir actuellement éliminer la possibilité d'un gène unique doué d'une action pléiotropique. Sur le plan pratique, l'exploration de cette zone chromosomique doit être proposée dans les lissencéphalies de type I, et sans doute aussi dans les formes incomplètes, telles que les lissencéphalies sans dysplasie faciale ou, à l'opposé, avec anomalies faciales sans troubles cérébraux sévères.

J.C. D.

1. McKusick VA. Mendelian Inheritance in Man, 8th ed. Baltimore: Johns Hopkins University Press, 1988.

2. Dobyns WB, Curry CJR, Hoyme HE, et al. Clinical and molecular diagnosis of MillerDieker syndrome. Am J Hum Genet 1991; 48 584-94.
Les surprises de la recombinaison homologue : rôle du protooncogène cellulaire $c$-src dans le développement osseux. Le gène $c$-src code pour une protéine kinase impliquée dans la transformation tumorale de certains tissus. Sa phosphorylation au cours de la mitose suggérait qu'elle pouvait jouer un rôle dans le contrôle du cycle cellulaire normal et plus généralement dans la croissance et la différenciation. Son expression élevéc dans le système nerveux et les plaquettes laissait penser que son intervention était particulièrement importante au niveau de ces tissus. Pour tester ces hypothèses, Soriano et al. (Houston, TX, USA) [1] ont $m / s n^{\circ} 5$, vol. 7, mai 91 obtenu, par recombinaison homologuc, des lignées de souris homozygotes pour une mutation "perte de fonction " du gène $c$-src. Contre toute attente, les sourcis src- sont atteintes d'une forme majeure d'ostéopétrose entraînant leur mort dans les premic̀res semaines de vie. Aucune anomalie n'a pu être détectéc dans le cerveau et les plaquettes de ces animaux, où l'on peut supposer que l'expression d'autres gènes de la famille des kinases apparentécs à $c$-src (yes, fyn, $h c k, f r g$, lyn ou blk) suppléent l'absence de la protéine Src. Cette suppléance fonctionnelle peut aussi expliquer l'absence de perturbation globale du cycle cellulaire. En revanche, les ostéoclastes, clérivés des monocytes circulants, ne contiendraient pas d'activité kinasique capable de remplacer celle normalement assurée par Src. Il est à noter que l'ostéopétrose murine liće à la mutation op est due à une anomalie du facteur stimulant la croissance des colonies de macrophages (M-CSF), dont le récepteur, codé par le gène $c$-fms, est aussi une protéine kinase. Si l'on ne peut affirmer la relation entre les mutations op et src, on peut faire l'hypothèse qu'elles perturbent une cascade de transmissions du signal commune aux protéines Fms et Src.

[1. Soriano P, et al. Cell 1991; 64 693-702.] 